\title{
Correction to: Nitrogen application and different water regimes at booting stage improved yield and 2-acetyl-1-pyrroline (2AP) formation in fragrant rice
}

Zhaowen Mo ${ }^{1,2}$, Yanhong $\mathrm{Li}^{1,3}$, Jun Nie ${ }^{1,3}$, Longxin $\mathrm{He}^{1,2}$, Shenggang Pan ${ }^{1,2}$, Meiyang Duan ${ }^{1,2}$, Hua Tian ${ }^{1,2}$, Lizhong Xiao ${ }^{1,2}$, Keyou Zhong ${ }^{1,2}$ and Xiangru Tang ${ }^{1,2^{*}}$

Correction to: Rice (2019) 12:74

https://doi.org/10.1186/s12284-019-0328-4

It was highlighted that the original article (Mo et al. 2019) contained an error in Fig. If which revealed the biosynthesis pathway of 2AP. This Correction article shows the correct Fig. 1 and incorrect Fig. 1. The original article has been updated.

Correct Fig. 1

Author details

'Department of Crop Science and Technology, College of Agriculture, South China Agricultural University, Guangzhou 510642, Guangdong, China.

${ }^{2}$ Scientific Observing and Experimental Station of Crop Cultivation in South China, Ministry of Agriculture.P. R. China, Guangzhou 510642, China.

${ }^{3}$ Agro-innovative Demonstration Base Guangdong Academy of Agricultural Sciences, Guangzhou 510642, China.

Incorrect Fig. 1

\footnotetext{
*Correspondence: tangxr@scau.edu.cn
}

The original article can be found online at https://doi.org/10.1186/s12284019-0328-4

'Department of Crop Science and Technology, College of Agriculture, South China Agricultural University, Guangzhou 510642, Guangdong, China ${ }^{2}$ Scientific Observing and Experimental Station of Crop Cultivation in South China, Ministry of Agriculture.P. R. China, Guangzhou 510642, China Full list of author information is available at the end of the article 


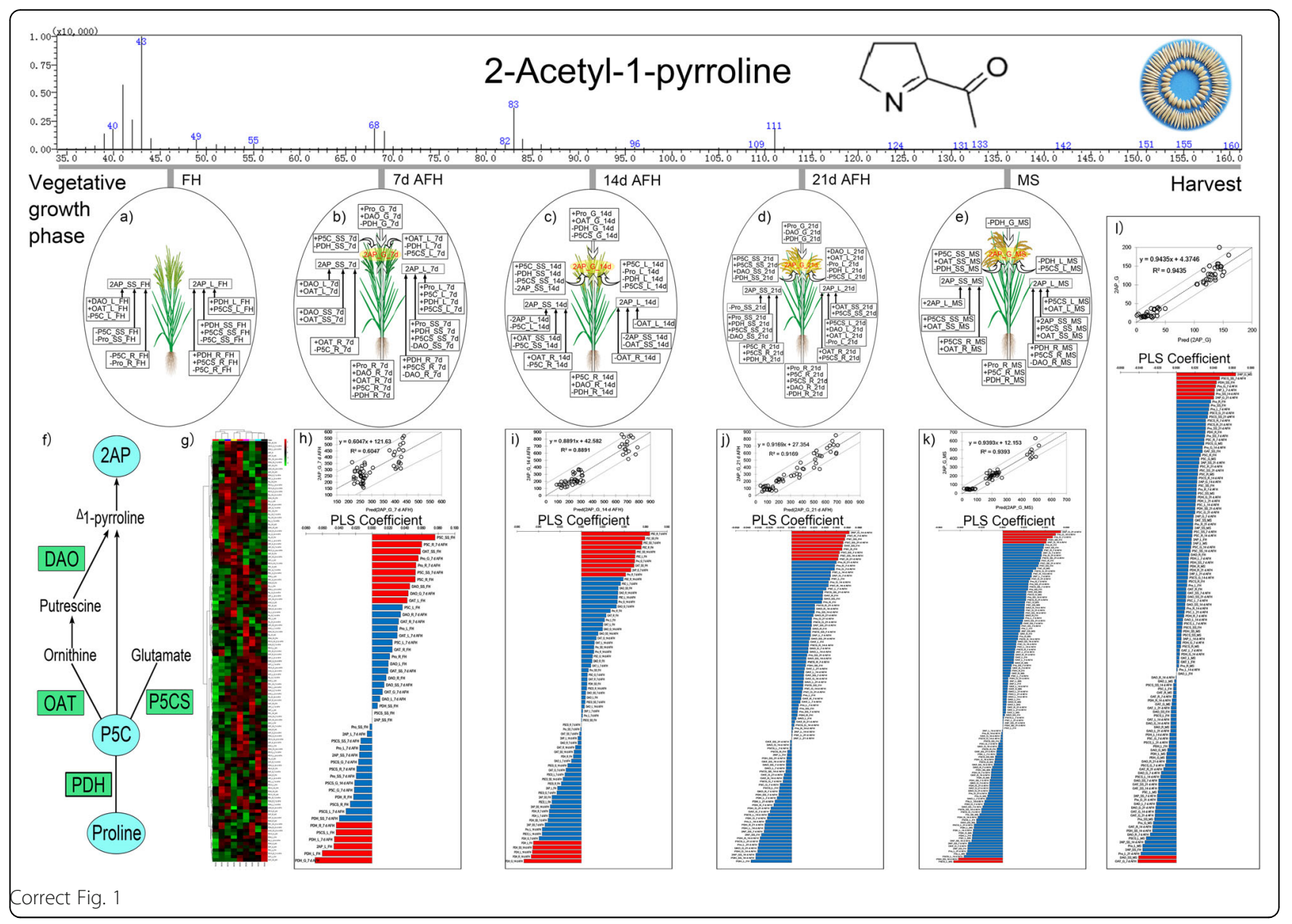




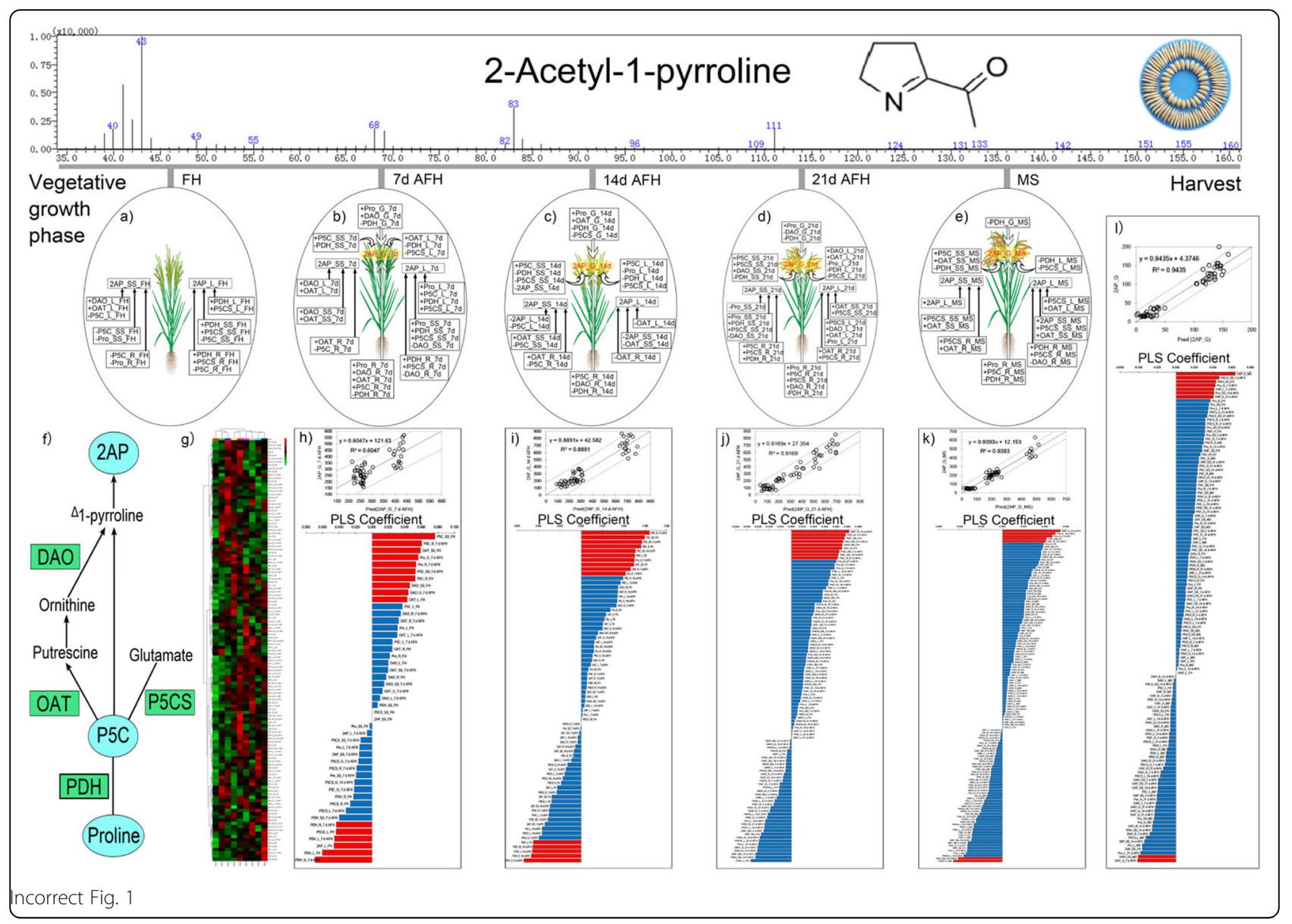

Published online: 31 October 2019

\section{Reference}

Mo Z et al (2019) Nitrogen application and different water regimes at booting stage improved yield and 2-acetyl-1-pyrroline (2AP) formation in fragrant rice. Rice 12:74. https://doi.org/10.1186/s12284-019-0328-4 